\title{
A phase 3 study of rituximab biosimilar HLX01 in patients with diffuse large B-cell lymphoma
}

Yuankai Shi ${ }^{*}$, Yongping Song ${ }^{2}$, Yan Qin ${ }^{1}$, Qingyuan Zhang ${ }^{3}$, Xiaohong Han ${ }^{1}$, Xiaonan Hong ${ }^{4}$, Dong Wang ${ }^{5}$, Wei Li ${ }^{6}$, Yang Zhang ${ }^{7}$, Jifeng Feng ${ }^{8}$, Jianmin Yang ${ }^{9}$, Huilai Zhang ${ }^{10}$, Chuan Jin ${ }^{11}$, Yu Yang ${ }^{12}$, Jianda Hu ${ }^{13}$, Zhao Wang ${ }^{14}$, Zhengming Jin ${ }^{15}$, Hang Su ${ }^{16}$, Huaqing Wang ${ }^{17}$, Haiyan Yang ${ }^{18}$, Weijun Fu ${ }^{19}$, Mingzhi Zhang ${ }^{20}$, Xiaohong Zhang ${ }^{21}$, Yun Chen ${ }^{22}$, Xiaoyan Ke ${ }^{23}$, Li Liü ${ }^{24}$, Ding Yü ${ }^{25}$, Guo'an Chen ${ }^{26}$, Xiuli Wang ${ }^{27}$, Jie Jin ${ }^{28}$, Tao Sun ${ }^{29}$, Xin $\mathrm{Du}^{30}$, Ying Cheng ${ }^{31}$, Pingyong $\mathrm{Yi}^{32}$, Xielan Zhao ${ }^{33}$, Chaoming Ma ${ }^{34}$, Jiancheng Cheng ${ }^{34}$, Katherine Chai ${ }^{34}$, Alvin Luk ${ }^{34}$, Eugene Liü ${ }^{34}$ and Xin Zhang ${ }^{34}$

\begin{abstract}
Rituximab in combination with chemotherapy has shown efficacy in patients with diffuse large B-cell lymphoma (DLBCL) for more than 15 years. HLX01 was developed as the rituximab biosimilar following a stepwise approach to demonstrate biosimilarity in analytical, pre-clinical, and clinical investigations to reference rituximab. With demonstrated pharmacokinetic similarity, a phase 3 multi-center, randomized, parallel, double-blind study (HLX01NHL03) was subsequently conducted to compare efficacy and safety between HLX01 plus cyclophosphamide, doxorubicin, vincristine, and prednisone ( $\mathrm{H}-\mathrm{CHOP}$ ) and reference rituximab plus CHOP (R-CHOP) in a total of 407 treatment-naïve, CD20-positive DLBCL patients aged 18-80 years. The primary efficacy endpoint was best overall response rate (ORR) within six cycles of treatment in the per-protocol set (PPS). Secondary endpoints included 1year efficacy outcomes, safety, and immunogenicity profile. The results showed difference in ORRs [H-CHOP 94.1\%; R-CHOP 92.8\%] between two treatment groups was 1.4\% (95\% confidence interval [Cl], -3.59 to 6.32, $p=0.608$ ) which falls within the pre-defined equivalence margin of $\pm 12 \%$. The safety profile was comparable between the treatment groups, with a similar overall incidence of treatment-emergent adverse events ( $\mathrm{H}-\mathrm{CHOP} 99.5 \%$, R-CHOP $99.0 \%, p=1.000$ ) and serious adverse events (H-CHOP 34.0\%, R-CHOP 32.5\%, $p=0.752$ ). This study established bioequivalence in efficacy and safety between HLX01 and reference rituximab. The trial was registered at http:// www.chinadrugtrials.org.cn on 26 August 2015 [\#CTR20150583].
\end{abstract}

Keywords: Rituximab biosimilar, DLBCL, Efficacy equivalence

\footnotetext{
* Correspondence: syuankai@cicams.ac.cn

${ }^{1}$ National Cancer Center/National Clinical Research Center for Cancer/Cancer

Hospital, Beijing Key Laboratory of Clinical Study on Anticancer Molecular

Targeted Drugs, Chinese Academy of Medical Sciences \& Peking Union

Medical College, Beijing, China

Full list of author information is available at the end of the article
}

(c) The Author(s). 2020 Open Access This article is licensed under a Creative Commons Attribution 4.0 International License, which permits use, sharing, adaptation, distribution and reproduction in any medium or format, as long as you give appropriate credit to the original author(s) and the source, provide a link to the Creative Commons licence, and indicate if changes were made. The images or other third party material in this article are included in the article's Creative Commons licence, unless indicated otherwise in a credit line to the material. If material is not included in the article's Creative Commons licence and your intended use is not permitted by statutory regulation or exceeds the permitted use, you will need to obtain permission directly from the copyright holder. To view a copy of this licence, visit http://creativecommons.org/licenses/by/4.0/. The Creative Commons Public Domain Dedication waiver (http://creativecommons.org/publicdomain/zero/1.0/) applies to the data made available in this article, unless otherwise stated in a credit line to the data. 
Treatment with rituximab, a monoclonal antibody against CD20, in combination with cyclophosphamide, doxorubicin, vincristine, and prednisone (R-CHOP) has been used in patients with diffuse large B-cell lymphoma (DLBCL) for more than 15 years with proven efficacy and safety [1]. With demonstrated highly similar analytical characterization and bioequivalence in pharmacokinetics and pharmacodynamics [2], we conducted this phase 3, multi-center, randomized, parallel, double-blind study (HLX01-NHL03) to establish the equivalence in clinical efficacy, safety, and immunogenicity between HLX01 plus CHOP (H-CHOP) and R-CHOP every 21 days for up to six cycles in treatment-naïve patients with CD20-positive DLBCL.

Eligible patients were treatment-naïve adults ( $\geq 18$ to $\leq 80$ years) with International Prognostic Index of $0-2$, clinical stages I-IV (Ann Arbor Staging) and histologically confirmed CD20-positive DLBCL. The primary efficacy endpoint was best overall response rate (ORR) within six cycles of treatment in the per-protocol set (PPS), and secondary efficacy endpoints included complete response rate, 1-year duration of response, 1year event-free survival, 1-year progression-free survival, 1-year disease-free survival, 1-year overall survival, and depletion of CD19-positive B-cells in peripheral blood.

From October 9, 2015 to March 10, 2017, a total of 560 patients were screened, of whom 407 patients were randomized (1:1) at 33 investigational sites; 361 patients (H-CHOP 173; R-CHOP 188) completed six cycles of treatment, and 328 patients (H-CHOP 157; R-CHOP 171) completed the study (Fig. 1a). Baseline characteristics are well balanced between two treatment groups (Fig. 1b). In the PPS, the best ORRs within six cycles of treatment in the PPS were $94.1 \%$ (95\% confidence interval $[\mathrm{CI}], 89.77$ to 97.04 ) and $92.8 \%$ (95\% CI, 88.19 to $96.00)$ in the $\mathrm{H}-\mathrm{CHOP}$ and $\mathrm{R}-\mathrm{CHOP}$ groups,

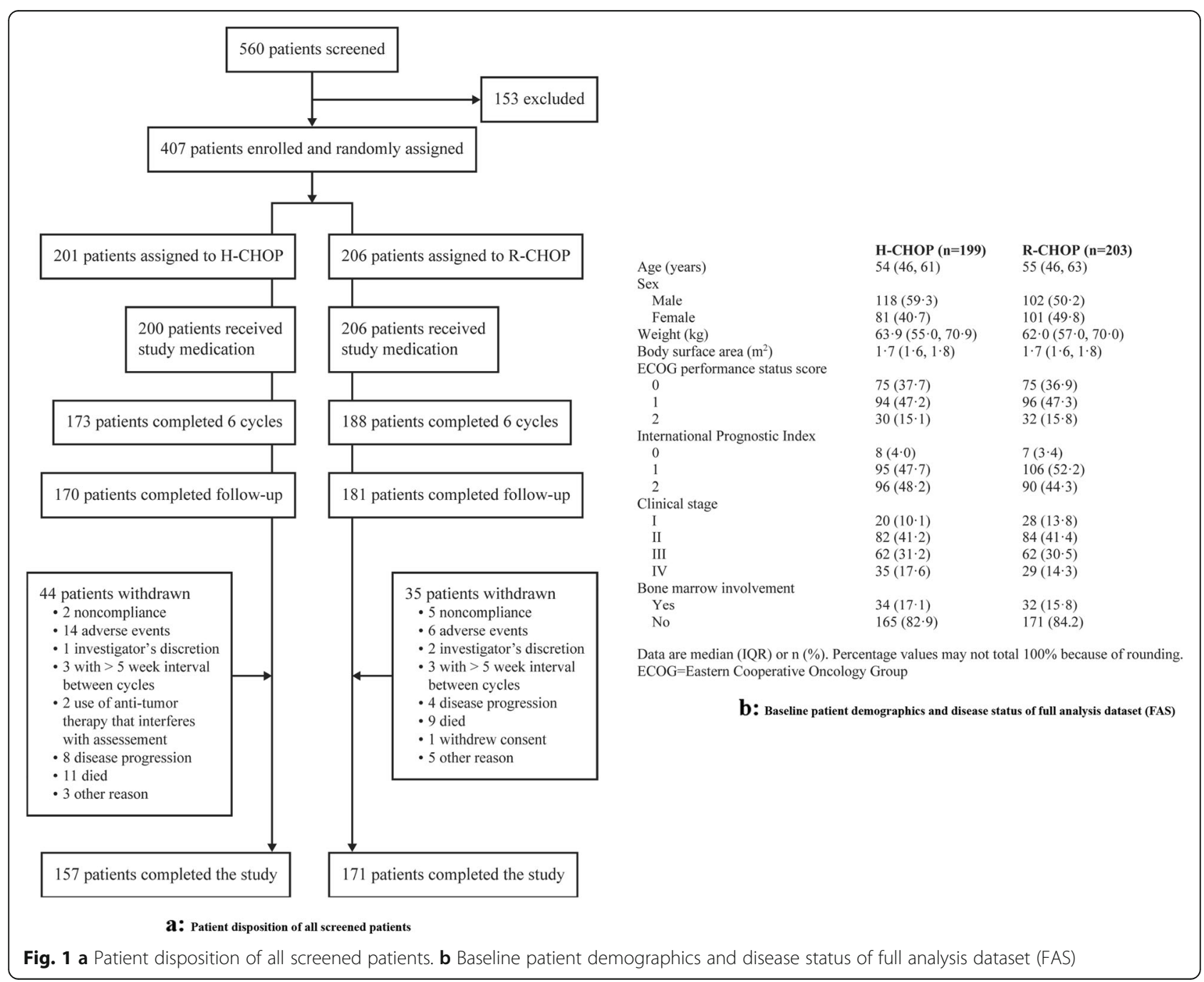


Table 1 Efficacy outcomes

\begin{tabular}{|c|c|c|c|c|c|c|}
\hline & \multicolumn{3}{|l|}{ Per-protocol dataset } & \multicolumn{3}{|l|}{ Full analysis dataset } \\
\hline & $\mathrm{H}-\mathrm{CHOP}(\boldsymbol{n}=188)$ & $\mathrm{R}-\mathrm{CHOP}(\boldsymbol{n}=194)$ & $\boldsymbol{P}$ value & $\mathrm{H}-\mathrm{CHOP}(\boldsymbol{n}=199)$ & $\mathrm{R}-\mathrm{CHOP}(\boldsymbol{n}=203)$ & $\boldsymbol{P}$ value \\
\hline Best overall response rate & $177(94 \cdot 1)$ & $180(92 \cdot 8)$ & 0.608 & $184(92.5)$ & $187(92 \cdot 1)$ & 0.839 \\
\hline Complete response & $88(46 \cdot 8)$ & $101(52 \cdot 1)$ & 0.231 & $90(45 \cdot 2)$ & $104(51 \cdot 2)$ & $0 \cdot 190$ \\
\hline Partial response & $89(47 \cdot 3)$ & $79(40 \cdot 7)$ & & $94(47 \cdot 2)$ & $83(40 \cdot 9)$ & \\
\hline Stable disease & $8(4 \cdot 3)$ & $13(6 \cdot 7)$ & & $11(5 \cdot 5)$ & $15(7.4)$ & \\
\hline Disease progression & $2(1 \cdot 1)$ & $1(0.5)$ & & $2(1 \cdot 0)$ & $1(0 \cdot 5)$ & \\
\hline No evidence of disease & $1(0.5)$ & 0 & & $2(1 \cdot 0)$ & 0 & \\
\hline \multicolumn{7}{|l|}{ Duration of response } \\
\hline Patients experiencing events & $25(13 \cdot 8)$ & $21(11 \cdot 5)$ & 0.424 & $26(13 \cdot 7)$ & $21(11 \cdot 1)$ & 0.355 \\
\hline Patients censored & $156(86 \cdot 2)$ & $161(88.5)$ & & $164(86 \cdot 3)$ & $168(88.9)$ & \\
\hline \multicolumn{7}{|l|}{ Event-free survival } \\
\hline Patients experiencing events & $80(42 \cdot 6)$ & $67(34.5)$ & $0 \cdot 125$ & $88(44 \cdot 2)$ & $71(35 \cdot 0)$ & 0.087 \\
\hline Patients censored & $108(57 \cdot 4)$ & $127(65 \cdot 5)$ & & $111(55 \cdot 8)$ & $132(65 \cdot 0)$ & \\
\hline 1-year event-free survival rate & $55.4(47.9,63.0)$ & $64 \cdot 5(57 \cdot 6,71 \cdot 4)$ & & $53 \cdot 7(46 \cdot 4,61 \cdot 0)$ & $63 \cdot 4(56 \cdot 6,70 \cdot 2)$ & \\
\hline \multicolumn{7}{|l|}{ Progression-free survival } \\
\hline Patients experiencing events & $31(16 \cdot 5)$ & $29(14.9)$ & 0.534 & $33(16 \cdot 6)$ & $30(14.8)$ & 0.473 \\
\hline Patients censored & $157(83.5)$ & $165(85 \cdot 1)$ & & $166(83.4)$ & $173(85 \cdot 2)$ & \\
\hline 1-year progression-free survival rate & $75 \cdot 0(66 \cdot 5,83 \cdot 6)$ & $80 \cdot 1(73 \cdot 5,86 \cdot 7)$ & & $74 \cdot 1(65 \cdot 6,82 \cdot 7)$ & $79.7(73 \cdot 1,86 \cdot 3)$ & \\
\hline \multicolumn{7}{|l|}{ Overall survival } \\
\hline Patient deaths & $15(8 \cdot 0)$ & $13(6 \cdot 7)$ & 0.661 & $16(8 \cdot 0)$ & $14(6 \cdot 9)$ & 0.701 \\
\hline Patients censored & $173(92 \cdot 0)$ & $181(93 \cdot 3)$ & & $183(92 \cdot 0)$ & $189(93 \cdot 1)$ & \\
\hline 1-year overall survival rate & $91.8(87.8,95 \cdot 8)$ & $92 \cdot 4(88 \cdot 3,96 \cdot 6)$ & & $91 \cdot 6(87 \cdot 6,95 \cdot 5)$ & $92 \cdot 1(88 \cdot 0,96 \cdot 3)$ & \\
\hline \multicolumn{7}{|l|}{ Disease-free survival } \\
\hline Patients experiencing events & $27(14.4)$ & $24(12 \cdot 4)$ & 0.462 & $28(14 \cdot 1)$ & $25(12 \cdot 3)$ & 0.477 \\
\hline Patients censored & $161(85 \cdot 6)$ & $170(87 \cdot 6)$ & & $171(85.9)$ & $178(87.7)$ & \\
\hline 1-year disease-free survival rate & $77.4(68.9,85.9)$ & $83.0(76 \cdot 7,89 \cdot 3)$ & & $76.9(68.4,85.4)$ & $82 \cdot 6(76 \cdot 2,88.9)$ & \\
\hline
\end{tabular}

Data are $\mathrm{n}(\%)$ or $\%,(95 \% \mathrm{Cl})$. Percentage values may not total $100 \%$ because of rounding

respectively, with an intergroup difference of 1.4\% (95\% CI, -3.59 to $6.32, p=0.608$ ). The efficacy equivalence between HLX01 and reference rituximab was demonstrated with $95 \%$ CIs falls entirely within the pre-defined margin of $\pm 12 \%$. The results of using the full analysis set (FAS) were consistent with the primary efficacy analysis in the PPS. Previous reports of R-CHOP in patients with DLBCL have shown ORRs ranging between 83\% and $88 \%[3,4]$, which is comparable with the result from this study. No significant differences were observed in the 1-year analysis of all secondary efficacy endpoints, in either the PPS or the FAS (Table 1).

The safety analysis set (Table 2) comprised 406 patients who received at least one treatment. 199/200 in H-CHOP group and 204/206 in R-CHOP group (HCHOP 99.5\%, R-CHOP 99.0\%, $p=1.000$ ) experienced at least one treatment-emergent adverse event; 68/200 in $\mathrm{H}-\mathrm{CHOP}$ and $67 / 206$ in R-CHOP (H-CHOP $34.0 \%$, RCHOP $32.5 \%, p=0.752$ ) experienced at least one serious adverse event; $14 / 200$ in $\mathrm{H}$-CHOP and $9 / 206$ in RCHOP (H-CHOP $7.0 \%$, R-CHOP $4.4 \%, p=0.252$ ) discontinued treatment because of adverse events (AEs). The most common AEs were hematological events such as decreased white blood cell count (H-CHOP 85.5\%; RCHOP 85.9\%), decreased neutrophil count (H-CHOP 79.0\%; R-CHOP 81.6\%), and anemia (H-CHOP 38.5\%; R-CHOP 35.0\%).

Among the patients observed with infusion-related reactions (IRRs), 61/200 in H-CHOP group and 61/206 in $\mathrm{R}-\mathrm{CHOP}$ group (H-CHOP 30.5\%; $\mathrm{R}-\mathrm{CHOP} 29.6 \%$ ), the most common reactions were those affecting skin and subcutaneous tissues. Most IRRs were grade 1 or 2 , and no grade 4 or 5 IRRs were reported. Increases in hepatitis B virus (HBV) DNA titer were observed in five patients in $\mathrm{H}$-CHOP group and eight patients in $\mathrm{R}-\mathrm{CHOP}$ group, and nine of whom were receiving antiviral therapy for chronic HBV; however, no patients developed signs or symptoms of fulminant hepatitis. 
Table 2 Safety profiles in the safety analysis dataset

\begin{tabular}{|c|c|c|}
\hline & $\mathrm{H}-\mathrm{CHOP}(\boldsymbol{n}=200)$ & $\mathrm{R}-\mathrm{CHOP}(\boldsymbol{n}=206)$ \\
\hline Patients with $\geq 1 \mathrm{TEAE}$ & $199(99.5)$ & $204(99 \cdot 0)$ \\
\hline Patients with $\geq 1$ SAE & $68(34.0)$ & $67(32.5)$ \\
\hline Patients with $\geq 1 \mathrm{AE}$ leading to treatment discontinuation & $14(7)$ & $9(4 \cdot 4)$ \\
\hline Patients deaths due to $\mathrm{AE}$ & $5(2 \cdot 5)$ & $3(1 \cdot 5)$ \\
\hline \multicolumn{3}{|l|}{ Adverse events with an incidence $\geq 10 \%$} \\
\hline \multicolumn{3}{|l|}{ Hematological } \\
\hline Decreased white blood cell count & $171(85 \cdot 5)$ & $177(85.9)$ \\
\hline Decreased neutrophil count & $158(79.0)$ & $168(81 \cdot 6)$ \\
\hline Anemia & $77(38.5)$ & $72(35 \cdot 0)$ \\
\hline Decreased platelet count & $34(17 \cdot 0)$ & $19(9 \cdot 2)$ \\
\hline Decreased lymphocyte count & $24(12 \cdot 0)$ & $34(16 \cdot 5)$ \\
\hline Decreased hemoglobin concentration & $23(11 \cdot 5)$ & $20(9 \cdot 7)$ \\
\hline \multicolumn{3}{|l|}{ Non-hematological } \\
\hline Nausea & $46(23 \cdot 0)$ & $49(23.8)$ \\
\hline Increased alanine aminotransferase & $49(24.5)$ & $38(18.4)$ \\
\hline Fever & $47(23.5)$ & $34(16 \cdot 5)$ \\
\hline Decreased appetite & $32(16 \cdot 0)$ & $42(20 \cdot 4)$ \\
\hline Increased lactate dehydrogenase & $30(15 \cdot 0)$ & $40(19 \cdot 4)$ \\
\hline Debilitation & $38(19.0)$ & $31(15 \cdot 0)$ \\
\hline Alopecia & $35(17.5)$ & $34(16 \cdot 5)$ \\
\hline Increased aspartate aminotransferase & $34(17.0)$ & $30(14 \cdot 6)$ \\
\hline Cough & $31(15 \cdot 5)$ & $26(12 \cdot 6)$ \\
\hline Vomiting & $22(11.0)$ & $30(14 \cdot 6)$ \\
\hline Upper respiratory tract infection & $19(9 \cdot 5)$ & $29(14 \cdot 1)$ \\
\hline Hypokalemia & $28(14 \cdot 0)$ & $17(8 \cdot 3)$ \\
\hline Constipation & $27(13.5)$ & $25(12 \cdot 1)$ \\
\hline Non-infectious pneumonia & $19(9 \cdot 5)$ & $24(11 \cdot 7)$ \\
\hline Pulmonary infection & $19(9 \cdot 5)$ & $24(11 \cdot 7)$ \\
\hline Diarrhea & $16(8 \cdot 0)$ & $22(10 \cdot 7)$ \\
\hline Chills & $20(10 \cdot 0)$ & $14(6 \cdot 8)$ \\
\hline \multicolumn{3}{|l|}{ Adverse events by CTCAE Grade } \\
\hline Grade 1 & $8(4 \cdot 0)$ & $6(2 \cdot 9)$ \\
\hline Grade 2 & $35(17 \cdot 5)$ & $35(17.0)$ \\
\hline Grade 3 & $54(27 \cdot 0)$ & $75(36 \cdot 4)$ \\
\hline Grade 4 & $98(49 \cdot 0)$ & $85(41 \cdot 3)$ \\
\hline Grade 5 & $4(2 \cdot 0)$ & $3(1.5)$ \\
\hline \multicolumn{3}{|l|}{ Grade 4 adverse events with an incidence $\geq 2.5 \%$} \\
\hline Decreased neutrophil count & $85(42 \cdot 5)$ & $75(36 \cdot 4)$ \\
\hline Decreased white blood cell count & $44(22 \cdot 0)$ & $42(20 \cdot 4)$ \\
\hline Febrile neutropenia & $5(2 \cdot 5)$ & $6(2 \cdot 9)$ \\
\hline Bone marrow failure & $5(2 \cdot 5)$ & $5(2 \cdot 4)$ \\
\hline
\end{tabular}

Data are $\mathrm{n}(\%)$. Percentage values may not total $100 \%$ because of rounding 
Anti-drug antibodies (ADAs) were detected in one patient $(<1 \%)$ in each treatment group at baseline and immediately before administration of the second treatment cycle. After 6 months of follow-up, ADAs were detected in one patient in $\mathrm{H}-\mathrm{CHOP}$ group and two patients in $\mathrm{R}$ CHOP group (H-CHOP 1.0\%, R-CHOP $1.7 \%, p=1.000$ ), and after 8 months of follow-up in seven patients in $\mathrm{H}$ $\mathrm{CHOP}$ group and six patients in R-CHOP group ( $\mathrm{H}-$ CHOP 7.1\%, R-CHOP 5.5\%, $p=0.629$ ). During the entire study, only one patient in R-CHOP group had both ADAs and neutralizing antibodies.

In conclusion, this study demonstrated therapeutic equivalence between HLX01 and reference rituximab. The analysis of the primary and secondary efficacy endpoints did not reveal any statistically significant differences between two treatment groups. The safety and immunogenicity profiles of HLX01 were comparable with reference rituximab with no clinically meaningful differences observed between two treatment groups.

\section{Abbreviations}

R-CHOP: Rituximab plus cyclophosphamide, hydroxydaunorubicin, oncovin (vincristine), and prednisone; DLBCL: Diffuse large B-Cell lymphoma; $\mathrm{H}$ CHOP: HLX01 plus cyclophosphamide, hydroxydaunorubicin, oncovin (vincristine), and prednisone; ORR: Best overall response rate; PPS: Perprotocol set; Cl: Confidence interval; FAS: Full analysis set; AE: Adverse event; IRR: Infusion-related reaction; HBV: Hepatitis B virus; ADA: Anti-drug antibody

\section{Acknowledgements}

We thank all the patients and families who were involved in the HLX01NHLO3 study and the clinical study teams and Hangzhou Tigermed Consulting Co., Ltd. for providing support for the study.

\section{Authors' contributions}

YKS was the lead principal investigator and contributed to the design and conception, interpretation data, and writing of the manuscript. YKS, YPS, YQ, QYZ, XHH, XNH, DW, WL, YZ, JFF, JMY, HLZ, CJ, YY, JDH, ZW, ZMJ, HS, HQW, HYY, WJF, MZZ, XHZ, YC, XYK, LL, DY, GAC, XLW, JJ, TS, XD, YC, PYY, and XLZ contributed to data collection. $\mathrm{XHH}$ contributed to detecting CD19- and CD20-positive B-cells in peripheral blood. JCC contributed to statistical data analysis and interpretation of the data. EL contributed to the design and conception of the research. CMM, AL, EL, and XZ contributed to the interpretation of data and the completeness and accuracy of the data. KC and $\mathrm{AL}$ provided writing assistance for the initial manuscript and editorial assistance for reviewing and editing subsequent versions of the manuscript. The authors read and approved the final manuscript.

\section{Funding}

The study was supported by the sponsor, Shanghai Henlius Biotech, Inc., and by grants from the China National Major Project for New Drug Innovation (Grant No. 2015ZX09501008, 2017ZX09304015) and the Chinese Academy of Medical Sciences (CAMS) Innovation Fund for Medical Sciences (CIFMS) (Grant No. 2016-I2M-1-001).

\section{Availability of data and materials}

The data that support the findings of this study are available from the corresponding author upon reasonable request.

\section{Ethics approval and consent to participate}

The protocol was developed by the sponsor of the study, Shanghai Henlius Biotech, Inc. The study protocol was reviewed and approved by the relevant independent ethics committee at each participating study center. Written informed consent was obtained from all study participants prior to screening. Data were collected by the site investigators who vouch for the completeness and accuracy of the data and the fidelity of the trial to the protocol. The sponsor analyzed the data. The study was conducted in accordance with the Declaration of Helsinki, Guideline for Good Clinical Practice, and applicable national and local regulations for clinical trials.

\section{Consent for publication}

Not applicable.

\section{Competing interests}

CMM, JCC, KC, AL, EL, and XZ are employees of Shanghai Henlius Biotech, Inc. All other authors declare no competing interests.

\section{Author details}

${ }^{1}$ National Cancer Center/National Clinical Research Center for Cancer/Cancer Hospital, Beijing Key Laboratory of Clinical Study on Anticancer Molecular Targeted Drugs, Chinese Academy of Medical Sciences \& Peking Union Medical College, Beijing, China. ${ }^{2}$ Affiliated Cancer Hospital of Zhengzhou University, Henan Cancer Hospital, Zhengzhou, China. ${ }^{3}$ Harbin Medical University Cancer Hospital, Harbin, China. ${ }^{4}$ Fudan University Shanghai Cancer Center, Shanghai, China. ${ }^{5}$ Daping Hospital, Third Affiliated Hospital of the Army Medical University, Chongqing, China. ${ }^{6}$ The First Bethune Hospital of Jilin University, Changchun, China. ${ }^{7}$ The Second Hospital of Dalian Medical University, Dalian, China. ${ }^{8}$ Jiangsu Cancer Hospital and Jiangsu Institute of Cancer Research, Nanjing, China. ${ }^{9}$ Changhai Hospital, Shanghai, China. ${ }^{10}$ Tianjin Medical University Cancer Institute \& Hospital, Tianjin, China. ${ }^{11}$ Cancer Center of Guangzhou Medical University, Guangzhou, China. ${ }^{12}$ Fujian Provincial Cancer Hospital, Fuzhou, China. ${ }^{13}$ Fujian Medical University Union Hospital, Fuzhou, China. ${ }^{14}$ Beijing Friendship Hospital, Capital Medical University, Beijing, China. ${ }^{15}$ The First Affiliated Hospital of Soochow University, Soochow, China. ${ }^{16}$ The 307th Hospital of Chinese People's Liberation Army, Beijing, China. ${ }^{17}$ Tianjin People's Hospital, Tianjin, China.

${ }^{18}$ Zhejiang Cancer Hospital, Hangzhou, China. ${ }^{19}$ Shanghai Changzheng Hospital, Shanghai, China. ${ }^{20}$ The First Affiliated Hospital of Zhengzhou University, Zhengzhou, China. ${ }^{21}$ The Second Affiliated Hospital of Zhejiang University School of Medicine, Hangzhou, China. ${ }^{22}$ Jinan Central Hospital Affiliated to Shandong University, Jinan, China. ${ }^{23}$ Peking University Third Hospital, Beijing, China. ${ }^{24}$ Tangdu Hospital, Air Force Medical University, Xi'an, China. ${ }^{25}$ Hubei Cancer Hospital, Wuhan, China. ${ }^{26}$ The First Affiliated Hospital of Nanchang University, Nanchang, China. ${ }^{27}$ The Second Hospital of Jilin University, Changchun, China. ${ }^{28}$ The First Affiliated Hospital, College of Medicine, Zhejiang University, Hangzhou, China. ${ }^{29}$ Liaoning Cancer Hospital \& Institute, Shenyang, China. ${ }^{30}$ Guangdong Provincial People's Hospital, Guangzhou, China. ${ }^{31}$ Jilin Cancer Hospital, Changchun, China. ${ }^{32}$ Hunan Cancer Hospital, Changsha, China. ${ }^{33}$ Xiangya Hospital, Central South University, Changsha, China. ${ }^{34}$ Shanghai Henlius Biotech, Inc., Shanghai, China.

Received: 11 February 2020 Accepted: 1 April 2020

Published online: 16 April 2020

\section{References}

1. Coiffier B, Lepage E, Brière J, Herbrecht R, Tilly H, Bouabdallah R, et al. CHOP Chemotherapy plus rituximab compared with $\mathrm{CHOP}$ alone in elderly patients with diffuse large-B-Cell lymphoma. The N Engl J Med. 2002;346(4):235-42.

2. Xu Y, Xie L, Zhang E, Gao W, Wang L, Cao Y, et al. Physicochemical and functional assessments demonstrating analytical similarity between rituximab biosimilar HLX01 and the MabThera®. MAbs. 2019;11(3):606-20.

3. Pfreundschuh $M$, Kuhnt E, Trümper L, Österborg A, Trneny M, Shepherd L, et al. CHOP-like chemotherapy with or without rituximab in young patients with good-prognosis diffuse large-B-cell lymphoma: 6-year results of an open-label randomised study of the MabThera International Trial (MInT) Group. Lancet Oncol. 2011;12(11):1013-22.

4. Pfreundschuh $M$, Truemper $L$, Gill D, Osterborg A, Pettengell R, Trneny M, et al. First analysis of the Completed Mabthera International (Mint) Trial in young patients with low-risk diffuse large B-cell lymphoma (DLBCL): addition of rituximab to a $\mathrm{CHOP}$-like regimen significantly improves outcome of all patients with the identification of a very favorable subgroup with $\mid \mathrm{PI}=0$ and no bulky disease. Blood. 2004;104(11):157.

\section{Publisher's Note}

Springer Nature remains neutral with regard to jurisdictional claims in published maps and institutional affiliations. 